\title{
PNEUMOTHORAX COMPLICATING THERAPEUTIC PNEUMOPERITONEUM
}

\author{
BY \\ N. F. CROFTS \\ From Kettlewell Hospital, Swanley, Kent
}

(RECEIVED FOR PUBLICATION APRIL 12, 1954)

Pneumothorax is a rare complication of artificial pneumoperitoneum. Thirty cases have been mentioned in the literature, but only 18 have been described in detail. Full descriptions have been given by Lumsden (1949), Yannitelli, Woodruff, Mueller, and Howard (1949), and Ross and Farber (1951). Jones and Yuill (1952) summarized 10 documented cases and added a case of their own. Since then reports of further single cases have been made by Wu and Neptune (1951), Epstein (1951), Nozzolli, Epstein, and Molnar (1952), Vysniauskas and Brueckner (1953) and Johnson (1954).

The cause of the complication and the path of escape of the air have been the chief points of discussion. The following description of yet another case is justified only because this time the point of entry of the air into the pleural cavity was observed through the thoracoscope.

\section{The Present Case}

A woman aged 33 years, a hospital orderly, was discovered to have tuberculosis of the right lung in August, 1950. She was kept under observation at a chest clinic until January 2, 1953, when she was admitted to Kettlewell Hospital because the disease had recently spread.

There were no symptoms apart from slight loss of weight and a mild productive cough. Her general condition was good. She had had a right-sided pes cavus and a high degree of myopia since early childhood. There were no other abnormal physical signs. She was afebrile and the pulse range was 70 to 80 per minute. The erythrocyte sedimentation rate was $17 \mathrm{~mm}$. in one hour (Westergren). Twelve sputum tests were negative for tubercle bacilli on direct examination and culture. The vital capacity was $1,600 \mathrm{ml}$.

Radiography showed tuberculous disease of moderate extent confined to the right upper lobe. Tomography did not demonstrate cavitation.

Streptomycin, 1 g., and sodium P.A.S., 20 g. daily, were given for 60 days. A pneumoperitoneum was induced with $600 \mathrm{ml}$. of air on January 20 . The needle was introduced just above the level of the umbilicus at the lateral border of the left rectus muscle. There was no surgical emphysema and the normal liver dullness was lost. The final pressure was $10 \mathrm{~cm}$. of water. On is the following day the first refill of $700 \mathrm{ml}$. was given $\mathbb{D}$ without preliminary fluoroscopy. The initial and final $\frac{0}{\mathbb{D}}$ pressures were $5 \frac{1}{2}$ and $7 \mathrm{~cm}$. respectively. Two days 3 later no air was seen under the diaphragm on fluoroscopy. No pneumorhorax was noted, but it may have been overlooked. A refill of $600 \mathrm{ml}$. was given and the initial and final pressures were 0 and $14 \mathrm{~cm}$. On fluoroscopy $₫$ immediately after this refill an air space was seen under the diaphragm. After the third refill fluoroscopy showed a right pneumothorax with atelectasis of the right upper lobe, but no air under the diaphragm. The $\bar{O}$ intra-pleural pressures were -3 to $0 \mathrm{~cm}$. of water, and $800 \mathrm{ml}$. of air was withdrawn from the pleural cavity $\stackrel{\mathbb{\Omega}}{\varrho}$ leaving the pressures at -10 to $-4 \mathrm{~cm}$. At no time $\overrightarrow{\vec{B}}$ since the induction of the pneumoperitoneum did the patient have pain or other symptoms to suggest that anything unusual had occurred. The pneumoperitoneum was abandoned and the pneumothorax was: maintained because it promised to be a satisfactory form of treatment.

On February 9 right thoracoscopy was performed $\stackrel{0}{x}$ under local anaesthesia by Mr. L. L. Whytehead. Immediately before the operation $850 \mathrm{ml}$. of air was introduced into the peritoneal cavity and the patient $ᄋ$ was kept lying down to prevent the air from escaping into the pneumothorax. At thoracoscopy the lung $\mathrm{O}$ appeared free and the pleura was healthy. An excellent view of the whole right diaphragm was obtained. The only abnormality at first detected was a small, oval, grey, pedunculated protuberance, $\frac{1}{2} \mathrm{~cm}$. wide by $1 \mathrm{~cm}$. N long, on the diaphragm just anterior to the paravertebral ${ }^{\circ}$ gutter. No opening could be seen in it. Physiological 0 saline solution $\left(200 \mathrm{ml}\right.$.) was then run into the pleural $\mathrm{\omega}_{\mathrm{N}}$ cavity and the patient was put into the sitting position. 0 A constant stream of air bubbles was seen issuing from a minute hole in the dome of the diaphragm whenever the saline overlapped it on inspiration. The hole was $\stackrel{\Phi}{+}$ so small that it would not have been noticed if the ? bubbles had not drawn attention to it. Its position ${ }^{\circ}$ was marked by a haemostatic clip fixed to the diaphragm $\underset{\mathbb{D}}{\stackrel{\circ}{*}}$ through the thoracoscope.

Radiographs after the operation showed the clip on $\stackrel{\mathbb{Q}}{\AA}$ the dome of the diaphragm $3 \mathrm{~cm}$. from the lateral chest $\overline{-}$ 

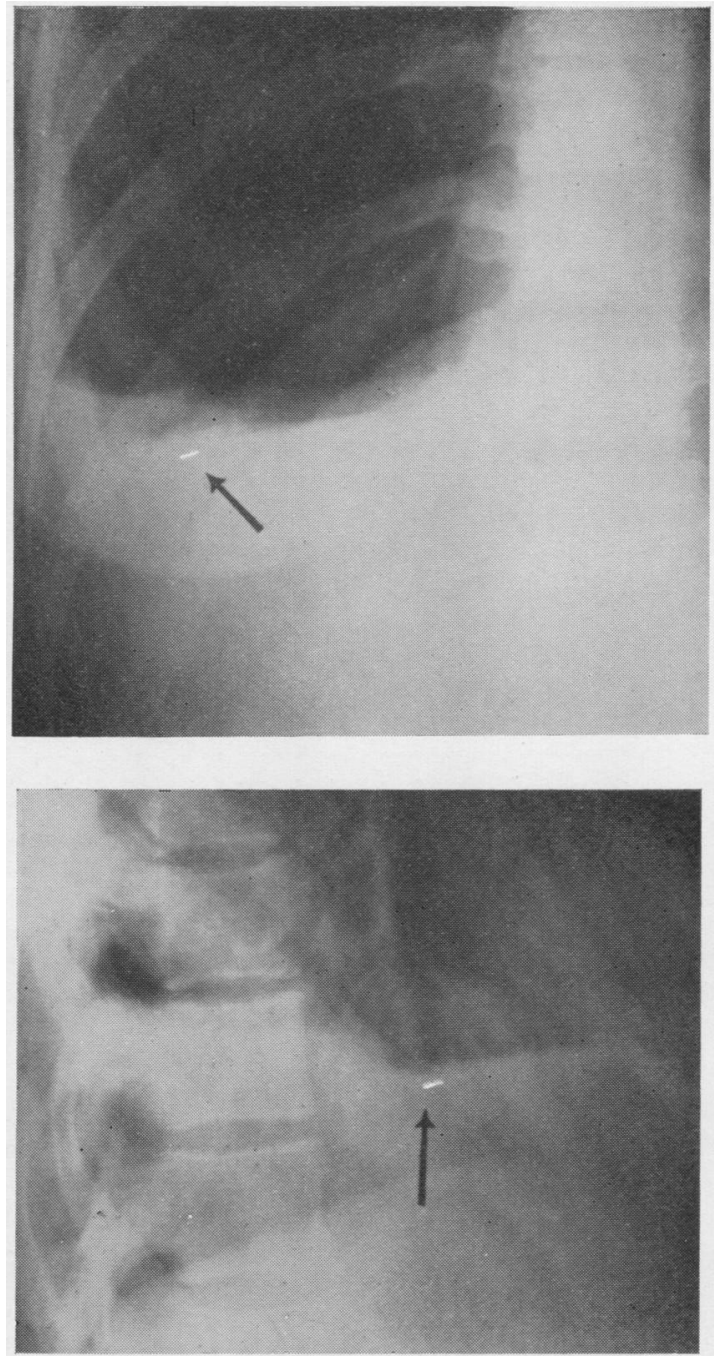

FIGs. $1 a$ and $b$.-Postero-anterior and right lateral radiographs taken the day after thoracoscopy. The arrows point to the clip which was inserted into the upper surface of the right hemidiaphragm at the site of the communication between the peritoneal and pleural cavities. The photographs have been touched up to make the clip more easily visible.

wall and $5 \mathrm{~cm}$. from the right border of the heart in the postero-anterior views. It lay $8 \mathrm{~cm}$. from the posterior chest wall and $9 \mathrm{~cm}$. from the anterior chest wall in the right lateral view (Figs. $1 a$ and $b$ ).

The progress of the patient was uneventful. The saline was rapidly absorbed from the pleural cavity. The right artificial pneumothorax was maintained, and the right upper lobe became re-aerated leaving a selective collapse of the diseased areas.

There was no escape of air from the pneumothorax into the peritoneal cavity. The patient was discharged from hospital on March 12.

\section{Discussion}

Several theories have been put forward to explain pneumothorax complicating therapeutic pneumoperitoneum.

Banyai and Jurgens (1940) and Simmonds (1946) considered that the air passes from the peritoneal cavity through one of the anatomical diaphragmatic hiatuses into the mediastinum and then ruptures the mediastinal pleura. This is not likely to be the correct explanation, because evidence of mediastinal emphysema is never present in these cases. Also, in four cases in addition to the one described here, a defect in the right diaphragm causing a direct communication between pleural and peritoneal cavities has been demonstrated either by thoracoscopy or at necropsy (Mellies, 1939 ; Smith, 1943 ; Yannitelli and others, 1949 ; Repa and Jacobson, 1951).

All authors who have discussed this condition since Simmonds's paper (1946) agree that there is a direct communication between the two serous cavities through the right diaphragm. Lumsden (1949) considered that most, if not all, of these cases are due to the abnormal persistence of a pleuro-peritoneal canal. $\mathrm{He}$ was not able to demonstrate this in his own patient, and at thoracoscopy the only feature of note was the presence of two sub-pleural air bubbles about 5 and $3 \mathrm{~mm}$. in diameter in the antero-lateral quadrant of the right diaphragm (Lumsden, 1954).

There are three reasons for rejecting the theory of a persisting pleuro-peritoneal canal, at least as a common cause of this complication. First, in six of these cases, sub-pleural blebs have been demonstrated on the diaphragm. If there were a persistent pleuro-peritoneal canal one would expect the air to pass directly into the pleural cavity without raising blebs on the diaphragmatic pleura. Secondly, a pleuro-peritoneal canal has not been demonstrated in any one of these cases, although in six the pleural cavity has been inspected with the thoracoscope, and in two necropsies have been performed. Thirdly, in those cases, including the present case, where the site of the opening has been demonstrated it did not lie at the expected site of a pleuro-peritoneal canal, namely, medially and posteriorly near to the oesophagus.

Sub-pleural air blebs on the right diaphragm were seen at thoracoscopy by Laird (1945) in four patients with pneumoperitoneum and right artificial pneumothorax. They lay mainly on the dome of the diaphragm, but in one patient they were studded practically all over the diaphragmatic surface (Laird, 1954). Laird suggested that rupture of 
one of these blebs is responsible when air from a pneumoperitoneum escapes into the pleural cavity, and that the blebs are the result of thinning of the diaphragmatic muscle following phrenic paralysis.

Spontaneous pneumothorax with simultaneous loss of air from pneumoperitoneum has been described on the left side only once, and in this case a pneumothorax developed on the right side also (Smith, 1943). The patient died, and at necropsy multiple small holes were found in both halves of the diaphragm. All other reported cases have been right-sided. The reason for this is unknown. Wu and Neptune (1951) suggest that it is due to the displacement of the liver by the pneumoperitoneum. This may be true, but is not supported by evidence. Johnson (1954) suggests that the pressure is greater under the right diaphragm during muscular exertion owing to a piston-like action of the liver. I have tested this point in three patients by measuring the intraperitoneal pressures under each half of the diaphragm simultaneously while the patient was exercising his legs in order to raise the intraabdominal pressure. There was no difference in pressure between right and left sides in any of these patients.

Jones and Yuill (1952) suggested that there are two types of defect underlying this complication, congenital and traumatic. The congenital type occurs immediately on induction of the pneumoperitoneum as in the cases described by Smith (1943) and Lumsden (1949). All other reported cases are said to be traumatic and occur as a result of continuous stretching of the diaphragm by the pneumoperitoneum. If this were true one would expect the complication most commonly in patients with a paralysed and atrophic hemidiaphragm due to phrenic nerve interruption, or with a long-standing pneumoperitoneum maintained at an unusually high pressure. In fact, these conditions do not usually exist. In the 10 cases which Jones and Yuill (1952) summarized only four had undergone phrenic nerve interruption and in one of these regeneration had taken place. Of five later cases none had a paralysed diaphragm on the affected side though two of them had been treated by phrenic interruption on the other side. It is submitted, therefore, that stretching of the diaphragm is not important.

It is concluded that most or all of these cases are due primarily to a congenital diaphragmatic defect caused by the lack of single or multiple muscle bundles. The peritoneum lying under the defect is forced through the gap by the raised intra-abdominal pressure and the overlying pleura is raised forming a bleb. Pneumothorax occurs only if the bleb ruptures. The interval between induction of the pneumoperitoneum and the onset of the pneumothorax depends mainly on the toughness of the pleura and peritoneum and the size of the muscle gap.

The features of the present case are consistent with this theory. Since an intra-peritoneal pressure of $5.5 \mathrm{~cm}$. was recorded before the first refil on the day after induction, it is probable that the air did not escape immediately after induction of the pneumoperitoneum, and that there was not a fully patent communication between the peritoneal and pleural cavities at this time. Nor could atrophy or much stretching of the diaphragm have taken place in the short time between the induction and the escape of air. As already stated, the patient had a right-sided pes cavus and a high degree of myopia since early childhood. The presence of these congenital defects provides some supporting evidence for the hypothesis that there was a congenital defect of the diaphragm. The small protuberance, observed at thoracoscopy, on the diaphragm just anterior to the paravertebral gutter was probably a sub-pleural bleb. A bleb was not seen at the site of the opening, but it has been shown experimentally by Macklin (1937) that pleural blebs are very difficult to identify after their rupture.

\section{SUMMARY}

A case of pneumothorax complicating a therapeutic pneumoperitoneum is described.

The site of the communication between abdomen and thorax was localized with the thoracoscope.

The cause of this complication is discussed.

1 wish to thank Dr. Paul Forgacs for many helpful suggestions and Mr. L. L. Whytehead for his thoracoscopy report.

\section{REFERENCES}

Banyai, A. L., and Jurgens, G. H. (1940). Amer. Rev. Tuberc., 42, 688.

Epstein, I. (1951). Dis. Chest, 20, 327.

Johnson, J. H. P. (1954). Brit. J. Tuberc., 48, 56.

Jones, J. Spencer, and Yuill, K. B. (1952). Ibid., 46, 30.

Laird, R. (1945). Tubercle, 26, 149.

Laird, R. (1945). Tubercle, 26, 149 .

Lumsden, E. G. Sita (1949). Thorax, 4, 147

(1954). Personal communication.

Macklin, C. C. (1937). Canad. med. Ass. J., 36, 414

Mellies, C. J. (1939). J. Mo. med. Ass., 36, 430.

Nozzoli, F., Epstein, L., and Molnar, L. (1952). Amer. Rev. Tuberc., 66,90 .

Repa, J. J., and Jacobson, H. R. (1951). Ibid., 63, 587.

Ross, J., and Farber, J. E. (1951). Ibid., 63, 67.

Simmonds, F. A. H. (1946). Lancet, 1, 530 .

Simith, C. N. (1943). Brit. med. J., 2, 404.

Smith, C. N. (1943). Brit. med. J., 2, 404.

Vysniauskas, C.̈ and Brueckner, H. H. (1953). Dis. Chest, 23, 572. Yannitelli, S. A., Woodruff, C. E., Mueller, E. E., and Howard, W. L. $Q$ (1949). Ibid., 60, 794. 